\title{
Ultrasonographic Findings of Subcutaneous
} Angioleiomyomas in the Extremities Based on Pathologic Subtypes

\author{
Dong Gun Kim, $M D^{1}$, Sun Joo Lee, MD, PhD ${ }^{1}$, Hye Jung Choo, MD, PhD ${ }^{1}$, Sung Kwan Kim, MD, \\ Jang Gyu Cha, MD, PhD², Hee Jin Park, MD, PhD ${ }^{3}$, Jong Won Kwon, MD, PhD ${ }^{4}$, Tae Eun Kim, MD $^{5}$, \\ Soo-Jin Jung, MD, $\mathrm{PhD}^{6}$
}

Departments of ${ }^{1}$ Radiology and ${ }^{6}$ Pathology, College of Medicine, Inje University Busan Paik Hospital, Busan 47392, Korea; ${ }^{2}$ Department of Radiology, College of Medicine, Soonchunhyang University Bucheon Hospital, Bucheon 14584, Korea; ${ }^{3}$ Department of Radiology, Sungkyunkwan University School of Medicine, Kangbuk Samsung Hospital, Seoul 03181, Korea; ${ }^{4}$ Department of Radiology, Dongcheon Dongkang Hospital, Ulsan 44495, Korea; ${ }^{5}$ Department of Radiology, Daegu Fatima Hospital, Daegu 41199, Korea

Objective: The purpose of this study was to describe the ultrasonographic findings of angioleiomyoma based on pathological subtypes.

Materials and Methods: Thirty-nine patients with subcutaneous angioleiomyomas in the extremities were retrospectively reviewed by two radiologists and a pathologist. Sonographic images were analyzed to evaluate each tumor's anatomic location, size, shape, margin, heterogeneity, echogenicity, associated findings, and vascularity.

Results: Angioleiomyomas were divided into 3 subtypes: capillary $(n=16)$, venous $(n=22)$, and cavernous $(n=1)$. The one cavernous angioleiomyoma was a hypoechoic mass with rich vascularity. Hypoechogenicity was more frequently observed for venous tumors $(77.3 \%)$ than for capillary tumors $(43.8 \%)$, and isoechogenicity was more frequently observed for capillary tumors $(56.2 \%)$ than for venous tumors $(22.7 \%)$. Moderate vascularity was more frequently observed for venous tumors $(59.1 \%)$ than for capillary tumors $(12.5 \%)$, and little vascularity was more frequently observed for capillary tumors $(62.5 \%)$ than for venous tumors $(13.6 \%)$. The aforementioned findings including echogenicity $(p=0.034)$ and vascularity $(p=0.003)$ were statistically significant.

Conclusion: Awareness of sonographic findings of angioleiomyomas based on pathologic subtypes could be helpful for diagnosing angioleiomyoma and could increase diagnostic accuracy for superficial soft-tissue masses in our practice.

Keywords: Angioleiomyoma; Extremity; Ultrasonography; Pathologic correlation; Pathologic type

\section{INTRODUCTION}

Angioleiomyomas are relatively rare benign smooth muscle tumors that arise from the tunica media of veins and arteries $(1,2)$. These tumors clinically manifest as small, movable, and painful subcutaneous masses that most commonly occur in the lower extremities. These masses are more likely to occur in females, and their peak incidence is between the fourth and sixth decades of life $(2,3)$.

Histologically, angioleiomyomas are divided into three subtypes based on World Health Organization criteria: capillary (or solid), venous, and cavernous tumors. Capillary tumors are

Received May 8, 2017; accepted after revision February 10, 2018.

This study was supported by 2016 Inje University Busan Paik Hospital research grant.

Corresponding author: Sun Joo Lee, MD, PhD, Department of Radiology, College of Medicine, Inje University Busan Paik Hospital, 75

Bokjiro, Busanjin-gu, Busan 47392, Korea.

- Tel: (8251) 890-6579•Fax: (8251) 896-1085•E-mail: sunjulee98@naver.com

This is an Open Access article distributed under the terms of the Creative Commons Attribution Non-Commercial License (https:// creativecommons.org/licenses/by-nc/4.0) which permits unrestricted non-commercial use, distribution, and reproduction in any medium, provided the original work is properly cited. 
characterized by numerous compacted smooth muscle bundles that are small in size and have slit-like vascular channels. Venous tumors have non-compacted smooth muscle bundles and vascular channels with thick muscular walls. Cavernous tumors are composed of dilated vascular channels with a small quantity of smooth muscle $(4,5)$.

Although different pathologic patterns can often be observed in the same tumor, one of the histological subtypes predominates (4). However, because diverse proportions of different components may result in various gray-scale and color Doppler imaging findings, the sonographic features of angioleiomyomas with respect to each subtype have not been thoroughly described in a detailed manner. The purpose of this study was to describe the sonographic findings of angioleiomyomas based on pathologic subtypes.

\section{MATERIALS AND METHODS}

Institutional Review Board approval was obtained, and informed consent was waived. This study involved 39 patients with pathologically proven angioleiomyoma who were diagnosed during the 10-year period from January 2005 to December 2014 at 9 institutions. All ultrasonography examinations were performed at 9 institutions using iU22 US systems (Philips Healthcare, Bothell, WA, USA), an ATL HDI 5000 system (Philips Healthcare), and a high-frequency linear transducer (7-12 MHz).

Imaging findings for each lesion were retrospectively reviewed by two musculoskeletal radiologists with 9 and 14 years of experience. The discordant findings between the two readers were subsequently resolved by consensus review. Images obtained via gray-scale sonography and color Doppler imaging were evaluated to determine each tumor's anatomic location, size, shape, margin, heterogeneity, echogenicity, associated findings, and vascularity.

Evaluations of anatomic location included whether the mass was located in the upper or lower extremities and whether it was located in the dermis, subcutaneous fat, or fascia. The size was determined by the maximum diameter of the mass in millimeters. Lesion shape was classified as round or oval. The margin of the mass was considered to be welldefined if more than two-thirds of the margin was clearly delineated from the surrounding tissue and partially defined if less than one-third of the margin was clearly delineated. With respect to heterogeneity, a tumor was considered homogeneous if there was uniform internal echotexture; otherwise, it was deemed heterogeneous. Echogenicity was classified as hypoechoic, isoechoic, and hyperechoic relative to findings for subcutaneous fat. Associated findings included observations of feeding vessels and calcification. Vascularity was considered rich if vascular signals were observed in more than $50 \%$ of the lesions, moderate if vascular signals were observed in $10-50 \%$ of the lesions, and little if vascular signals were observed in less than $10 \%$ of the lesion (including cases in which a vascular signal was absent). All patients underwent excision of their masses, which were subjected to pathologic analysis. A review of specimens to classify them into pathologic angioleiomyoma subtypes was conducted by a pathologist blinded to the sonographic findings.

The recorded data were analyzed using SPSS version 14.0 software (SPSS Inc., Chicago, IL, USA). Age and size correlations among angioleiomyoma subtypes were examined using Mann-Whitney $\mathrm{U}$ test. The categorical variables were compared using Fisher's exact test (location, shape, margin, and associated findings) or chi-square test (sex, heterogeneity, echogenicity, and vascularity) depending on the expected frequencies in each cell. $A$ $p$ value less than 0.05 was considered to be statistically significant. Interobserver agreement in the evaluation of ultrasound images was calculated using the $\kappa$ statistic, with $\kappa$ values of 0.20 or less, $0.21-0.40,0.41-0.60,0.61-0.80$, and $0.81-1.00$ indicating poor, fair, moderate, good, and excellent interobserver agreement, respectively.

\section{RESULTS}

The study group was composed of 20 males and 19 females (mean age, 46 years; age range, 27-73 years). All patients complained of a palpable mass in their extremities (with 12 masses in the upper extremities and 27 masses in the lower extremities) and all of the masses were located in subcutaneous fat layers.

The 39 lesions were divided into 3 types: capillary (11 lesions in males and 5 lesions in females), venous ( 9 lesions in males and 13 lesions in females), and cavernous (1 lesion in a female).

The one cavernous tumor was a well-defined, homogeneous, hypoechoic mass (approximately $6 \mathrm{~mm}$ ) with rich vascularity and several adjacent feeding vessels in the 1st web space of the left hand (Fig. 1). Angioleiomyomas of the other two subtypes were mostly in the lower extremities (in $75 \%$ and $68.2 \%$ of cases for capillary and 


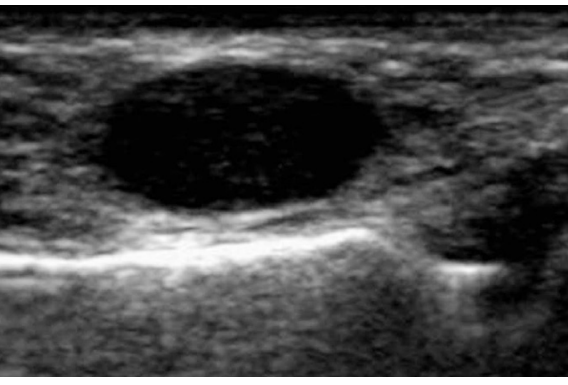

A

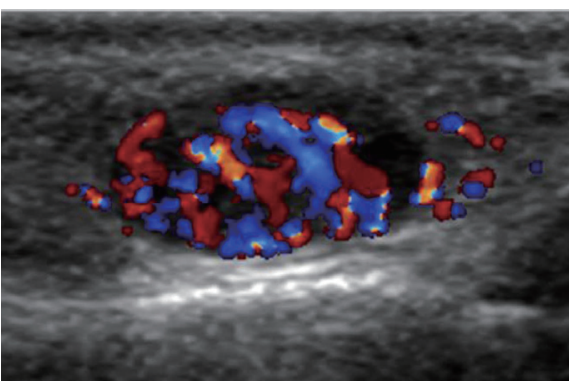

B

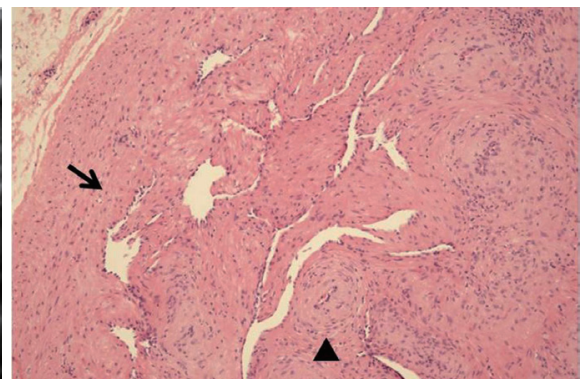

C

Fig. 1. 35-year-old female with palpable mass that was confirmed to be cavernous angioleiomyoma in 1st web space of left hand. A. Gray-scale sonogram in longitudinal plane shows well-defined, homogeneously hypoechoic mass. B. Color Doppler sonogram of same lesion shows abundant vascularity with several feeding vessels. C. Photomicrograph shows that this mass had dilated vascular channels with minimal smooth muscle that merged with surrounding smooth muscles (arrow) and was mixed with thick-walled vessels (arrow head), indicating that tumor was cavernous angioleiomyoma (original magnification, x 100, H\&E).

venous tumors, respectively), although this tendency was not statistically significant. Age, sex, and mean size did not significantly differ between the capillary and venous subtypes. The patients' demographic data for capillary and venous types of angioleiomyomas are summarized in Table 1.

A comparison of sonographic findings for capillary and venous angioleiomyomas is shown in Table 2. Hypoechogenicity was more frequently observed for venous tumors (17 of 22; 77.3\%) than for capillary tumors (7 of $16 ; 43.8 \%$ ) (Fig. 2). In contrast, isoechogenicity was more frequently observed for capillary tumors ( 9 of $16 ; 56.2 \%$ ) than for venous tumors (5 of 22; 22.7\%) (Fig. 3).

Rich vascularity was similarly prevalent for capillary (4 of $16 ; 25 \%$ ) and venous ( 6 of $22 ; 27.3 \%$ ) tumors. Moderate vascularity was more frequently observed for venous tumors (13 of 22; 59.1\%) than for capillary tumors (2 of 16; $12.5 \%$ ) (Fig. 2), and little vascularity was more frequently observed for capillary tumors (10 of $16 ; 62.5 \%$ ) than for venous tumors (3 of $22 ; 13.6 \%$ ) (Fig. 3 )

The echogenicity $(p=0.034)$ and vascularity $(p=0.003)$ differences between capillary and venous angioleiomyomas were statistically significant. Capillary and venous angioleiomyomas did not significantly differ with respect to shape, margin, heterogeneity, or associated findings (feeding vessels and calcification). Interobserver agreement was almost perfect for analyses of all of the sonographic features of angioleiomyomas. Kappa ranged from 0.855 (echogenicity and vascularity) to 1.000 (margin and calcifications).

\section{DISCUSSION}

Angioleiomyomas are rare smooth muscle tumors originating from the tunica media of veins and arteries.
Table 1. Comparison of Demographic Data, Location and Size of Angioleiomyomas between Capillary- and Venous-Types

\begin{tabular}{|c|c|c|c|}
\hline & $\begin{array}{l}\text { Capillary Type } \\
\quad(n=16)\end{array}$ & $\begin{array}{l}\text { Venous Type } \\
(n=22)\end{array}$ & $P$ \\
\hline $\operatorname{Sex}(\%)^{*}$ & & & 0.272 \\
\hline Female & $5(31.2)$ & $13(59.1)$ & \\
\hline Male & $11(68.8)$ & $9(40.9)$ & \\
\hline $\mathrm{Age}^{\dagger}$ & $48(36-67)$ & $49(34-70)$ & 0.965 \\
\hline Location $(\%)^{*}$ & & & 0.647 \\
\hline Upper extremities & $4(25)$ & $7(31.8)$ & \\
\hline Lower extremities & $12(75)$ & $15(68.2)$ & \\
\hline Size $(m m)^{\dagger}$ & $13.5(5-53)$ & $11.7(5-26)$ & 0.919 \\
\hline
\end{tabular}

*Data are No. (\%) of cases, ${ }^{\dagger}$ Data are median (range) of cases.

Hachisuga et al. (6) reviewed 562 cases of angioleiomyoma and reported that the peak of incidence was in the fourth to sixth decades of life, that these tumors predominantly occurred in females (female:male ratio, 1.7:1), and that $89 \%$ of the examined cases involved tumors in the extremities (with $75 \%$ of these tumors in the lower extremities). In our series, the ratio of male to female was nearly 1. Twenty-seven of 39 tumors (69.2\%) occurred in the lower extremities, and the others occurred in the upper extremities.

Many prior studies have reported that pain is a major clinical feature of angioleiomyomas (6); such pain appears to be caused by the active contraction of smooth muscles, which results in local ischemia (7). Unfortunately, we could collect clinical records regarding pain or tenderness of the lesion from only 21 out of our 39 patients. Thirteen of them $(62 \%)$ presented with pain or tenderness elicited by light touch or a change in temperature.

Angioleiomyomas can be found anywhere in the body and can develop in the dermis, subcutaneous fat, or fascia (8). Furthermore, Zhang et al. (9) reported that subcutaneous 
Table 2. Comparison of Sonographic Findings for Angioleiomyomas between Capillary- and Venous-Types

\begin{tabular}{|c|c|c|c|c|}
\hline Sonographic Findings & Capillary Type $(n=16)$ & Venous Type $(n=22)$ & $P$ & Interobserver Agreement \\
\hline Shape $(\%)^{*}$ & & & 0.611 & 0.924 \\
\hline Round & $4(25)$ & $4(18.2)$ & & \\
\hline Oval & $12(75)$ & $18(81.8)$ & & \\
\hline Margin $(\%)^{*}$ & & & 0.235 & 1.000 \\
\hline Well-defined & $15(94)$ & $22(100)$ & & \\
\hline Partially-defined & $1(6)$ & $0(0)$ & & \\
\hline Heterogeneity $(\%)^{*}$ & & & 0.152 & 0.924 \\
\hline Homogeneous & $8(50)$ & $16(72.7)$ & & \\
\hline Heterogeneous & $8(50)$ & $6(27.3)$ & & \\
\hline Echogenicity (\%)* & & & 0.034 & 0.855 \\
\hline Hypoechoic & $7(43.8)$ & $17(77.3)$ & & \\
\hline Isoechoic & $9(56.2)$ & $5(22.7)$ & & \\
\hline Vascularity (\%)* & & & 0.003 & 0.855 \\
\hline Rich & $4(25)$ & $6(27.3)$ & & \\
\hline Moderate & $2(12.5)$ & $13(59.1)$ & & \\
\hline Little & $10(62.5)$ & $3(13.6)$ & & \\
\hline \multicolumn{5}{|l|}{ Associated findings (\%)* } \\
\hline Feeding vessel & $7(43.7)$ & $12(54.5)$ & 0.511 & 0.924 \\
\hline Calcifications & $2(12.5)$ & $0(0)$ & 0.088 & 1.000 \\
\hline
\end{tabular}

${ }^{*}$ Data are No. $(\%)$ of cases.

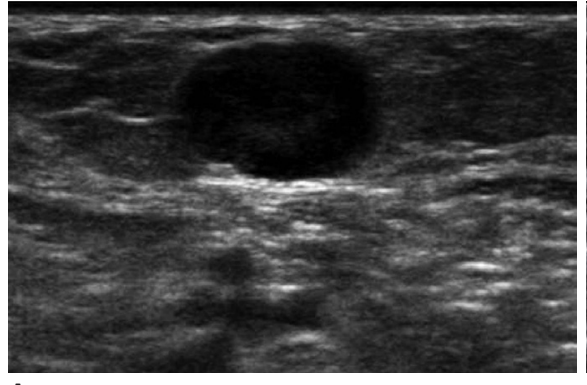

A

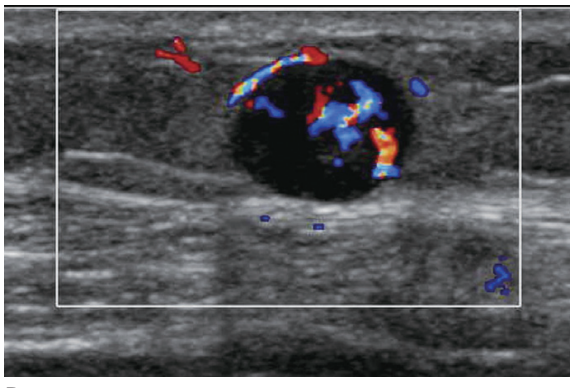

B

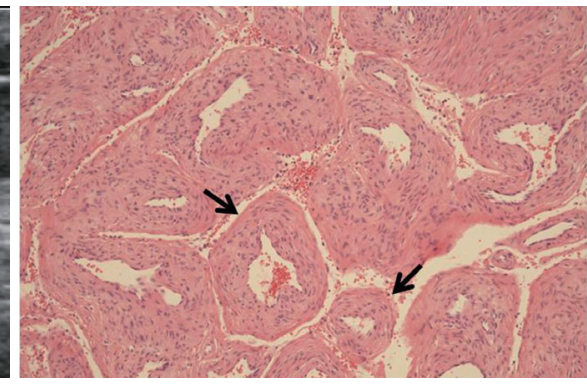

C

Fig. 2. 67-year-old female with palpable mass that was confirmed to be venous angioleiomyoma in 4th-5th palmar region of hand.

A. Gray-scale sonogram in longitudinal plane shows well-defined, homogeneously hypoechoic mass. B. Color Doppler sonogram of same lesion shows moderate vascularity with several feeding vessels. C. Photomicrograph shows that this mass had vessels (arrows) with thick muscular walls that merged with smooth muscle bundles, indicating that tumor was venous angioleiomyoma (original magnification, x 100, H\&E).

angioleiomyomas were more commonly detected in the superficial location of the subcutaneous fat layer, close to or adjoining the dermis. In our series, all of the tumors were superficially located in the subcutaneous fat layer, as well.

The ultrasonographic findings of angioleiomyoma are well-established in the literature and include an oval shape, a well-defined margin, a homogeneous echotexture, hypoechogenicity, and moderate or high vascular density (3, $5,10)$. In our study, angioleiomyomas were also frequently well-defined, oval-shaped, homogeneous, and hypoechoic. When comparing the gray-scale sonographic findings between venous type and capillary type angioleiomyomas, hypoechogenicity was significantly frequently found in the venous type, whereas isoechogenicity was present in the capillary type. Previous studies have demonstrated that angioleiomyomas appear as hypervascular masses on color Doppler imaging. Gomez-Dermit et al. (5) reported that vascular density was moderate or high in $50 \%$ of examined cases of angioleiomyoma and that blood flow was detected in all examined cases. In our study, moderate and rich vascularity was also more frequent, as we observed these features in 25 of 39 tumors (64.1\%). When comparing the color Doppler findings between venous and capillary types, venous angioleiomyomas typically appeared as hypervascular masses, as observed in previous studies, whereas capillary 


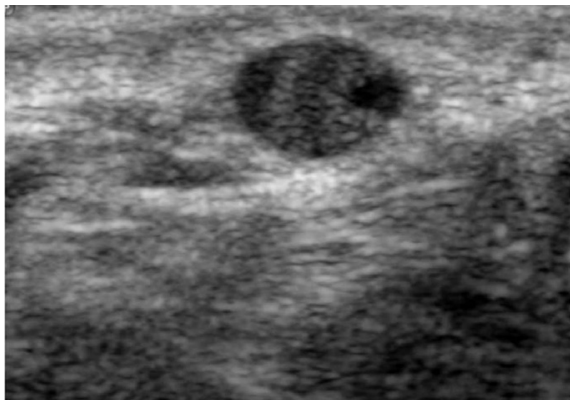

A

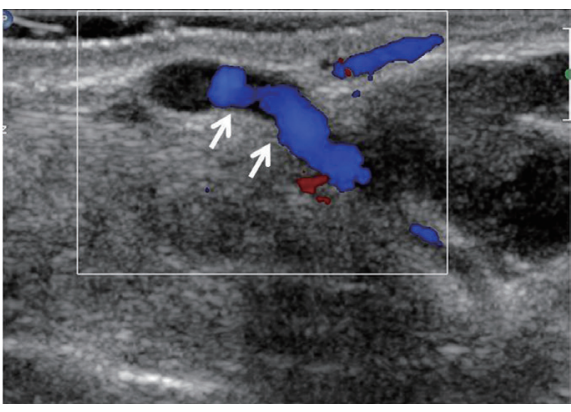

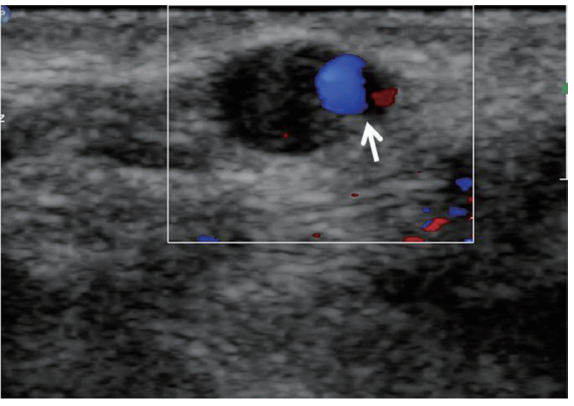

B

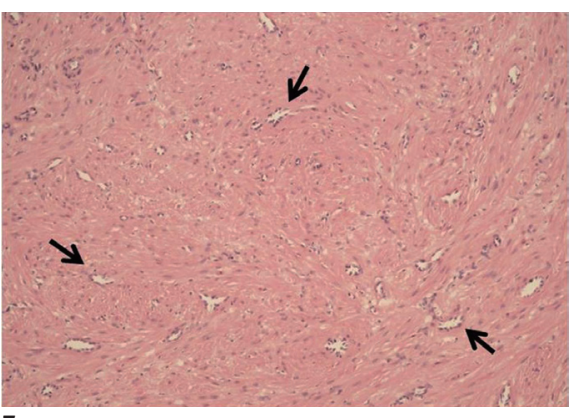

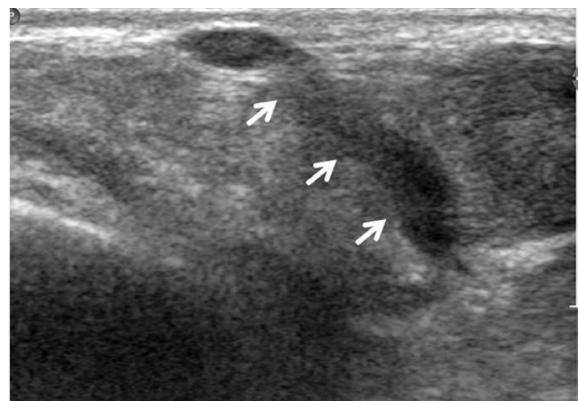

C

Fig. 3. 53-year-old female with palpable mass that was confirmed to be capillary angioleiomyoma in left calf.

A, B. Transverse scan of gray-scale and color Doppler sonogram show well-defined, homogeneously isoechoic mass with little vascularity and feeding vessel (arrow). C, D. Longitudinal scan of gray-scale and color Doppler sonogram show absence of vascularity in mass with linear feeding vessels (arrows). E. Numerous capillary-sized vessels (arrows) surrounded by closely packed smooth muscle bundles are visible, indicating that tumor was capillary angioleiomyoma (original magnification, x 100, H\&E).

tumors often exhibited little vascularity. It is not well known why the gray-scale and color Doppler findings were different between the capillary types and venous types. However, when considering that capillary angioleiomyomas are histologically composed of numerous capillary-sized vessels and closely packed smooth muscle bundles, resulting in the collapse of intratumoral vessels and a higher proportion of muscular components in the tumor, they are likely to be hypovascular masses and to show isoechogenicity similar to the muscle on ultrasonographic examination. On the other hand, because the venous type has internal, thick, muscularly walled-vessels and preservation of the vessel lumen, it is more likely to be hypervascular and hypoechoic than the capillary type. A prior study reported that capillary tumors are the most common type of angioleiomyomas (6). However, the results of our study suggest that the most common angioleiomyoma subtype is venous rather than capillary based on pathologic and imaging characteristics. Further study is needed to correlate between histology and ultrasonographic findings of angioleiomyoma.

Additionally, intratumoral calcifications were found in 2 of 33 angioleiomyomas (6.1\%) in our study, and both of them were capillary type. Calcification is known to be an uncommon ultrasonographic finding in angioleiomyomas.
In prior studies, it was considered to be a secondary degeneration because calcification was usually found in the larger angioleiomyomas $(5,6)$.

The differential diagnosis of angioleiomyomas can include many cutaneous and subcutaneous soft-tissue tumors, tumors of skin appendages, or mesenchymal origin tumors. Isoechoic masses with little vascularity can be commonly observed in a wide range of disease entities such as lipoma, epidermal cyst, foreign body granuloma, and organizing hematoma. Internal hyperechoic striations or hyperechoic dot-like foci make it easier to diagnose lipoma (fibrous septa) and epidermal cyst (keratin debris). Organizing hematoma and foreign body granuloma can display a more heterogeneous echotexture and frequently have associated findings (calcification, posterior shadowing). When diagnosing hypoechoic and hypervascular softtissue masses, hemangioma and non-subungual glomus tumor should be preferentially considered. Hemangioma commonly presents as a poorly defined mass without pain. Rich hypervascularity with diffusely scattered irregular vessels is commonly observed in non-subungual glomus tumors $(1,3,9,11)$. In the series reported by Park et al. (3), a specific tumor vascular pattern (several clustered linear vessels that converged at a single point) was a 
statistically significant finding for angioleiomyoma among hypervascular masses (hemangioma and non-subungual glomus tumor) as based on color Doppler imaging. In the English literature published to date, there are no reported specific sonographic features or differential diagnostic clues for atypical imaging features of angioleiomyomas, such as isoechoic masses with little vascularity. Although we cannot be certain, we propose that one of the associated findings, feeding vessels, may be helpful in narrowing down the wide array of differential diagnoses for subcutaneous isoechoic masses with little vascularity.

Our study had several limitations. First, this study involved a small number of cases, including only one case of cavernous angioleiomyoma. Therefore, we could not compare the three angioleiomyoma subtypes.

Second, it is known that power Doppler sonography has the advantage of improving evaluation of parenchymal vascularity, particularly in the low-flow state. However, only color Doppler images were analyzed in our study. Third, consensus among pathologists with respect to subdividing angioleiomyomas into three types was not achieved because of the multicenter study design. The last limitation was the retrospective nature of the study and the fact that the observers were not blinded to patients' diagnoses.

In conclusion, angioleiomyomas commonly present as homogeneously hypoechoic, well-defined, oval masses with hypervascularity on gray-scale sonography and color Doppler imaging. However, echogenicity and vascularity can be variable for angioleiomyomas depending on the pathologic subtype. In particular, isoechogenicity and little vascularity are more commonly seen in the capillary subtype than in the venous subtype. Awareness of these sonographic findings could be helpful for diagnosing angioleiomyoma and could increase diagnostic accuracy for superficial softtissue masses in our practice.

\section{REFERENCES}

1. Jin W, Kim GY, Park SY, Chun YS, Nam DH, Park JS, et al. The spectrum of vascularized superficial soft-tissue tumors on sonography with a histopathologic correlation: Part 1, benign tumors. AJR Am J Roentgenol 2010;195:439-445

2. Gupte C, Butt SH, Tirabosco R, Saifuddin A. Angioleiomyoma: magnetic resonance imaging features in ten cases. Skeletal Radiol 2008;37:1003-1009

3. Park HJ, Kim SS, Lee SY, Choi YJ, Chung EC, Rho MH. Sonographic appearances of soft tissue angioleiomyomas: differences from other circumscribed soft tissue hypervascular tumors. J Ultrasound Med 2012;31:1589-1595

4. Fletcher C. D. M, Unni K. K, Mertens F. Pathology and Genetics of Tumours of Soft Tissue and Bone, 1st ed. Lyon: IARC Press, 2002:128-129

5. Gomez-Dermit V, Gallardo E, Landeras R, Echevarría F, García Barredo R. Subcutaneous angioleiomyomas: gray-scale and color Doppler sonographic appearances. J Clin Ultrasound 2006;34:50-54

6. Hachisuga T, Hashimoto H, Enjoji M. Angioleiomyoma. A clinicopathologic reappraisal of 562 cases. Cancer 1984;54:126-130

7. Yoo HJ, Choi JA, Chung JH, Oh JH, Lee GK, Choi JY, et al. Angioleiomyoma in soft tissue of extremities: MRI findings. AJR Am J Roentgenol 2009;192:W291-W294

8. Freedman AM, Meland NB. Angioleiomyomas of the extremities: report of a case and review of the Mayo Clinic experience. Plast Reconstr Surg 1989;83:328-331

9. Zhang JZ, Zhou J, Zhang ZC. Subcutaneous angioleiomyoma: clinical and sonographic features with histopathologic correlation. J Ultrasound Med 2016;35:1669-1673

10. Smith J, Wisniewski SJ, Lee RA. Sonographic and clinical features of angioleiomyoma presenting as a painful Achilles tendon mass. J Ultrasound Med 2006;25:1365-1368

11. Hwang JW, Ahn JM, Kang HS, Suh JS, Kim SM, Seo JW. Vascular leiomyoma of an extremity: MR imaging-pathology correlation. AJR Am J Roentgenol 1998;171:981-985 